\title{
Introduction: Dealing with Big Data-Lessons from Cognitive Computing
}

\author{
Ahsan Abdullah $^{1}$ - Amir Hussain ${ }^{2} \cdot$ Imtiaz Hussain Khan $^{1}$
}

Published online: 20 November 2015

(c) Springer Science+Business Media New York 2015

Big Data analytics is an emerging area of fast-growing importance as it provides innovative ways to efficiently analyse large and complex data effectively. Many approaches have been developed to extract meaningful information and knowledge from large multidimensional data sets involving high veracity, volume, velocity, and value. Unlike traditional approaches, cognitive computing systems are not based on predetermined answers or actions to perform Big Data analytics; instead, they are trained using efficient, scalable, natural, and biologically inspired approaches, including computational intelligence and machine learning algorithms without compromising their sophistication and performance. Cognitive computing systems interact with, learn naturally from people and Big data, and thus help increase the productivity of what either humans or machines could do on their own.

This Special Issue is aimed at promoting multidisciplinary research, particularly in the complementary areas of cognitive psychology, biology, and computing science for dealing with Big Data challenges across these domains. Accordingly, the articles selected in this Special Issue

Ahsan Abdullah

aabdullah1@kau.edu.sa

Amir Hussain

ahu@cs.stir.ac.uk

Imtiaz Hussain Khan

ihkhan@kau.edu.sa

1 Faculty of Computing and Information Technology, King Abdulaziz University, P. O. Box 80200, Jeddah, Kingdom of Saudi Arabia

2 Division of Computing Science and Mathematics, School of Natural Sciences, University of Stirling, Stirling, Scotland, UK report a range of cognitively inspired computational approaches to deal with different aspects of Big Data analytics. Following a rigorous peer-review process, ranging from three-to-four rounds of revisions; five articles have been accepted for publication. The reviews of the final article co-authored by the guest editors were handled independently by another editorial board member.

In the first article presented in this Special Issue, $\mathrm{Wu}$, Pang, and Coghill propose an integrative qualitative and quantitative modelling framework for inferring biochemical systems that could help, especially biologists, better understand natural biochemical systems. The proposed framework demonstrates how the identification of biochemical systems can be performed and evolved in an integrative manner by reusing, composing, and evolving biochemical modules qualitatively, and by mutating kinetic rates quantitatively.

In the next article, Du et al. propose a novel computational method to detect specific biomarkers for different groups of cancer types. The proposed method identifies specific biomarkers for a given cancer group based on different factors, including the same survival rates, the same type, and grade. The proposed methodology is thoroughly evaluated using eight cancer types and a number of benchmark microarray gene expression data sets from public databases.

In the next article, Lin et al. describe a cognitively inspired psycho-linguistically motivated approach to build a quiz generation system, which combines similarity information derived from linked data and Resource Description Framework (RDF) resources. The authors effectively exploit this similarity information to gauge the difficulty of quizzes, confirming that a higher similarity among concepts leads to a more difficult quiz formulation, and vice versa. The proposed methodology is evaluated 
using human subjects to demonstrate the validity of the semantic similarity-based clustering results.

In the next article, Bilal, Mujtaba, and Jaffar address the challenge of restoration of spatial degradations in imagesa complex problem, involving both uncertainty and large volume of data, in visual information processing. The authors propose a cognitively inspired computational solution, which effectively models the problem as a constraint-optimization problem and employs particle swarm optimization-based evolution to minimize the error for enhanced restoration of images.

Finally, Abdullah et al. describe a cognitively inspired integrative study to investigate the feasibility of using nearinfrared spectroscopy (NIRS) technology for studying the spatiotemporal activity of the two brain hemispheres in concept-colour association tasks. The authors employ a multichannel NIRS device to record channel-wise activation data for 10 male students; and cluster the data using an indigenous clustering technique to identify channels having similar spatiotemporal activity. Their results show that it is possible to associate human cognitive brain activities in the two hemispheres using NIRS and cognitively inspired clustering techniques.

The guest editors would thank the anonymous reviewers for their valuable and timely reviews of the manuscripts, which have helped ensure the quality of this Special Issue. 